\title{
Biosynthesis and characterictics of silver nanoparticles
} obtained using Saccharomyces cerevisiae M437

\section{Oksana Skrotska ${ }^{1}$, Yevhen Kharchenko' ${ }^{1}$, Yuliia Laziuka ${ }^{1}$, Andrii Marynin', Maksym Kharchuk ${ }^{2}$}

\author{
1 - National University of Food Technologies, Kyiv, Ukraine \\ 2 - Danylo Zabolotny Institute of Microbiology and Virology of the National \\ Academy of Sciences of Ukraine, Kyiv, Ukraine
}

Keywords:

Nanoparticles

Silver

Yeast

Biosynthesis

Saccharomyces

cerevisiae

Article history:

Received

18.03.2021

Received in revised

form 11.08.2021

Accepted

30.09.2021

Corresponding

author:

Oksana Skrotska

E-mail:

skrotska@ukr.net

DOI:

$10.24263 / 2304-$

974X-2021-10-3-14

\section{Abstract}

Introduction. Due to the wide antimicrobial spectrum, silver nanoparticles (AgNPs) have great potential for use in the food industry to control foodborne pathogens.

Materials and methods. The culture supernatant and cell-free aqueous extract from biomass Saccharomyces cerevisiae M437 were used for the synthesis of AgNPs. The fact of the synthesis of biogenic AgNPs was confirmed by analysing the absorption spectra of the samples in the range of 200-700 $\mathrm{nm}$. The size and zeta potential of AgNPs were determined using Zetasizer Nano ZS. The morphology of nanoparticles was examined using electron microscopy.

Results and discussion. Using spectral analysis in the UVvisible region, it was confirmed the formation of AgNPs in the investigated solutions. A pronounced absorption peak of AgNPs obtained using a cell-free aqueous extract from S. cerevisiae M437 was recorded in the wavelength range from 300 to $540 \mathrm{~nm}$ with a peak at $425 \mathrm{~nm}$. For nanoparticles obtained using the supernatant, a widening spectral range of absorption was observed, which may be associated with the aggregation of AgNPs.

AgNPs synthesized using the supernatant S. cerevisiae M437 had a spherical shape with a diameter of about $15 \mathrm{~nm}$. The polydispersity index (PdI) of AgNPs solutions was 0.3 , and the zeta potential was $13.6 \mathrm{mV}$. After storage for 45 days at $4{ }^{\circ} \mathrm{C}$, the $\mathrm{PdI}$ value increased 1.6 times, and the zeta potential increased by $11.7 \%$. This may indicates a possible change in the shape of AgNPs, the formation of an agglomerate, or other processes that takes place in a colloidal solution during storage.

AgNPs that were obtained using a cell-free aqueous extract from biomass of $S$. cerevisiae M437 had an oval shape with a size of $21.3 \times 14.2 \mathrm{~nm}$. The PdI and zeta potential values were similar to the nanoparticles obtained using the supernatant. However, after storage, these values differed significantly: the value of PdI increased 1.3 times, and the zeta potential decreased by $29 \%$. So, the solution of silver nanoparticles obtained in this way is more stable after storage under the specified conditions.

Conclusions. The possibility of extracellular synthesis of silver nanoparticles using the yeast Saccharomyces cerevisiae M437 has been shown. The shape, size, and zeta potential of biogenic AgNPs are described and their stability after storage is proved. 


\section{Introduction}

Silver nanoparticles (AgNPs) have great potential to be used in the food industry due to a wide range of antimicrobial activity against foodborne pathogens such as Listeria monocytogenes (Du et al., 2019; Amer et al., 2021), Campylobacter (Silvan et al., 2018), Vibrio parahaemolyticus, Escherichia coli, Salmonella typhimurium (Du et al., 2019; Chandhru et al., 2019), Staphylococcus aureus (Yahya et al., 2021; Reddy et al., 2021), Klebsiella pneumoniae (Huang et al., 2020), and fungi of the genus Aspergillus (Bocate et al., 2019).

AgNPs can be used for the manufacturing of packaging materials for food products nuts (Tavakoli et al., 2017), apricots (Shahat et al., 2020), strawberries (Oliveira et al., 2021), bell peppers (Kandasamy, 2020), shrimps (Paidari et al., 2021), poultry meat (Zhao et al., 2021), and pork (Kuuliala et al., 2015). Such packaging prolongs the shelf life of food and prevents the development of pathogenic bacteria (Sachdev et al., 2021).

The use of AgNPs in nanobiosensors allow increasing the sensitivity of detection in food and drinking water of bacterial pathogens such as Escherichia coli (Zhou et al., 2015; Qiao et al., 2021), Staphylococcus aureus (Gasparyan and Bazukyan, 2013; Hovhannisyan et al., 2017), and Salmonella typhimurium (Ma et al., 2021).

One of the potential uses of AgNPs is winemaking. Traditionally, sulfur dioxide is used as a preservative in winemaking, which poses certain risks for some groups of consumers. AgNPs, due to their antimicrobial action, reduce the use of sulfur dioxide in winemaking (Gil-Sanchez et al., 2019; Loira et al., 2020).

Another area of application of AgNPs in the food industry is their use as nanocatalysts, in particular, to accelerate the decomposition of starch due to the immobilization of $\alpha$ amylase on the surface of AgNPs (Ernest et al., 2012; Krishnakumar et al., 2018).

Given the wide range of applications for AgNPs in the food industry, the demand for AgNPs is also growing. At the same time, the problems of economic and environmentally safe synthesis of AgNPs remain unresolved. There are various methods for the synthesis of AgNPs: chemical and physical methods, as well as biogenic synthesis. Due to a number of disadvantages inherent in the chemical and physical synthesis of nanoparticles - the use of aggressive, toxic and expensive reagents, high synthesis temperature or pressure, negative impact on the environment (Lekha et al., 2021; Halder et al., 2021), based on the above, the biological synthesis of nanoparticles provides promising alternative (Gaurav et al., 2019).

Plants, filamentous fungi, bacteria and yeast cells can be used for biogenic synthesis of nanoparticles (Kumar et al., 2021). The choice of each of these objects for the biosynthesis of nanoparticles has its advantages and disadvantages. Among the disadvantages of using plants are the use of large areas for their cultivation, the duration of growth, the cost of collecting plants and the extraction of biomolecules involved in the synthesis of nanoparticles. (Castillo-Henriquez et al., 2020). The main disadvantage in usage of filamentous fungi is in the long cultivation time (Bahrulolum et al., 2021). The use of bacteria compared to plants and fungi has a number of advantages. But it should be noted that bacteria are prokaryotes, so the products of their metabolism will be less biocompatible compared to eukaryotic models. Bacteria lack a system of capping and polyadenylation that protect biomolecules and nanoparticles from degradation and formation of toxic compounds in the body (Liu et al., 2021). Among the advantages of using yeast compared to bacteria is that yeast, unlike most bacteria, is simpler and safer to work with, since it does not require specific biosafety measures (Grasso, 2020).

Depending on the choice of biological producer, it's cultivation conditions, as well as the parameters of biogenic synthesis, AgNPs of different sizes and shapes can be formed. 
That will affect their further biological properties and applicability in the food industry. That is why the purpose of our work was to study the synthesis of silver nanoparticles using the culture fluid supernatant and a cell-free aqueous extract of the yeast Saccharomyces cerevisiae M437 and to check the stability after storage.

\section{Materials and methods}

\section{Culture media and chemicals}

Medium 1. Saburo medium (dextrose $-40.0 \mathrm{~g} / \mathrm{l}$, bacteriological agar - $15.0 \mathrm{~g} / \mathrm{l}$, a mixture (1:1) of fermented animal tissue and pancreatic casein hydrolysate $-10.0 \mathrm{~g} / \mathrm{l}, \mathrm{pH}=$ 5.6). This medium was used to store and passivate the culture of S. cerevisiae M437 (in test tubes on agar slant).

Medium 2. YPD medium (glucose - $20.0 \mathrm{~g} / \mathrm{l}$, peptone - $20.0 \mathrm{~g} / \mathrm{l}$, yeast extract $10.0 \mathrm{~g} / \mathrm{l})$. This medium was used for the cultivation of S. cerevisiae M437 for further biosynthesis of silver nanoparticles.

Silver nitrate salt $\left(\mathrm{AgNO}_{3}, 99.99 \%\right)$ Sigma-Aldrich (Steinheim, Germany).

\section{Cultivation of Saccharomyces cerevisiae M437}

The culture of S. cerevisiae M437 from the collection of live cultures of the Department of Biotechnology and Microbiology of the National University of Food Technologies was maintained in a test tubes on agar slant at $4{ }^{\circ} \mathrm{C}$. To prepare the inoculum, the culture was washed from the surface of the agar slant (the amount of inoculum with a titer of $10^{4}$ to $10^{5}$ cells $/ \mathrm{ml}$ was $5 \%$ of the volume of the medium), and added to $150 \mathrm{ml}$ of sterile YPD medium. Cultivation was carried out in $750 \mathrm{ml}$ flasks with $150 \mathrm{ml}$ of medium at $30^{\circ} \mathrm{C}, 200 \mathrm{rpm}$ for 24 hours. Cultivated until reaching $\mathrm{OD}_{600}=2$.

\section{Biosynthesis of silver nanoparticles}

Obtaining of supernatant. After 24 hours of the yeast culturing, the following procedure was performed: the culture fluid was centrifuged at $5.000 \mathrm{rpm}$ for $30 \mathrm{~min}$ to separate the cells. The supernatant was separated from the cells by pouring into a sterile flask. Obtained supernatant was filtered through a sterile $0.22 \mu \mathrm{m}$ syringe filter.

Obtaining of a cell-free aqueous extract. The culture fluid was centrifuged at 5000 rpm for 30 minutes. The supernatant was drained, and the cell pellet was washed three times from residual nutrient medium with sterile double-distilled water. The washed cells were resuspended in $150 \mathrm{ml}$ of sterile double-distilled water and incubated at $30{ }^{\circ} \mathrm{C}, 320 \mathrm{rpm}$ for 72 hours. After incubation, centrifugation was performed at $5000 \mathrm{rpm}$ for 30 minutes. The precipitate was separated and the supernatant was used for further studies as a cell-free extract. The obtained cell-free extract was filtered through a sterile syringe filter with a pore diameter of $0.22 \mu \mathrm{m}$.

Biosynthesis conditions. A solution of silver nitrate was added to the supernatant or water extract to a final concentration of $1 \mathrm{mM}$. The samples were kept at $45^{\circ} \mathrm{C}$ under static conditions for 72 hours. A $1 \mathrm{mM}$ aqueous solution of silver nitrate, a YPD nutrient medium supplemented with $1 \mathrm{mM}$ silver nitrate, an aqueous cell-free extract without the addition of silver nitrate solution, and a supernatant without the addition of silver nitrate solution were used as control samples. The control samples were incubated under the same conditions as the experimental ones. 
Isolation of silver nanoparticles was performed as follows: centrifugation at $14.000 \mathrm{rpm}$ for $20 \mathrm{~min}$, the supernatant was drained, and the precipitate of nanoparticles was washed with deionized water. These steps were repeated three times (Jalal et al., 2018).

\section{UV-vis spectroscopy of synthesized silver nanoparticles}

The absorption spectra of the samples were measured using a UV-Vis (Thermo Spectronic UV300, Spectronic Unicam, England) spectrophotometer in the wavelength range of 200-700 nm (Hashim et al., 2020). The measurements were carried out in quartz cuvettes. Absorbance was measured with a resolution of $2 \mathrm{~nm}$. Measurements were carried out 24, 48, and $72 \mathrm{~h}$ after the addition of silver nitrate to the test samples (cell-free aqueous extract, supernatant). Double distilled water was used as a blank experiment.

\section{Analysis of the silver nanoparticles size and zeta potential}

Analysis of nanoparticle's size were performed by determining the hydrodynamic diameter (HD) (Foujdar et al., 2021). The HD was monitored by dynamic light scattering (DLS) using a two-angle particle and molecular size analyzer Zetasizer Nano ZS (Malvern, UK). We performed all measurements at a constant temperature $\left(25^{\circ} \mathrm{C}\right)$ in a neutral medium $(\mathrm{pH}=7.0)$ three times. For statistical calculations, we used Stat Plus Pro 5.9.8 software. Software and STATISTICA, version 8.0 (StatSoft, Inc. 2007).

\section{Electron microscopy}

Nanoparticle sizes and their general morphology were determined by electron microscopy (Kthiri et al., 2021). To achieve this, we prepared alcohol suspensions of nanoparticles, dried them at room temperature, and applied to copper grids with a carbon coating. Subsequently, we analyzed the samples using a transmission electron microscopy (JEM-1400 Jeol, Japan) at an accelerating voltage of $80 \mathrm{kV}$ and an instrumental magnification of $50000-100000 x$.

\section{Statistical analysis}

All results are presented as the median of the values with interquartile range - Me [LQ $\mathrm{UQ}$ ], where $\mathrm{Me}=$ median $(50 \%$ percentile), $\mathrm{LQ}=25 \%$ percentile and $\mathrm{UQ}=75 \%$ percentile. We tested the null hypothesis using the nonparametric Mann-Whitney test and the Wilcoxon matched pairs test (WMP-test). The difference between the compared groups was considered statistically significant at a value of $p<0.05$ (Fay and Malinovsky, 2017). Calculations of median values with interquartile range with Microsoft Office Excel, 2019, and all statistical calculations - with Stat Plus Pro 5.9.8 software. Software and STATISTICA, version 8.0 were provided (StatSoft, Inc. 2007).

\section{Results and discussion}

\section{Synthesis of Silver Nanoparticles and UV-Vis Spectral Analysis}

After the addition of silver nitrate to the cell-free aqueous extract of S. cerevisiae M437, the color of the reaction mixture began to change from transparent to light brown and turned dark brown until the end of biosynthesis $\left(72 \mathrm{~h}\right.$ at $45^{\circ} \mathrm{C}$ under static conditions) (Figure 1, A). When silver nitrate was added to the supernatant of $S$. cerevisiae M437 culture liquid, the 
color of the reaction mixture at the end of biosynthesis changed from light brown to almost black (Figure 1, B). The color change is the first evidence of a successful biogenic synthesis of silver nanoparticles (Kthiri et al., 2021). The indicated color change of the reaction mixture (cell-free aqueous extract, supernatant) upon addition of silver nitrate is explained by the excitation of surface plasmon resonance (SPR), which indicates a decrease in silver ions $\left(\mathrm{Ag}^{+}\right)$and their bioreduction to AgNPs (Rosman et al., 2020).
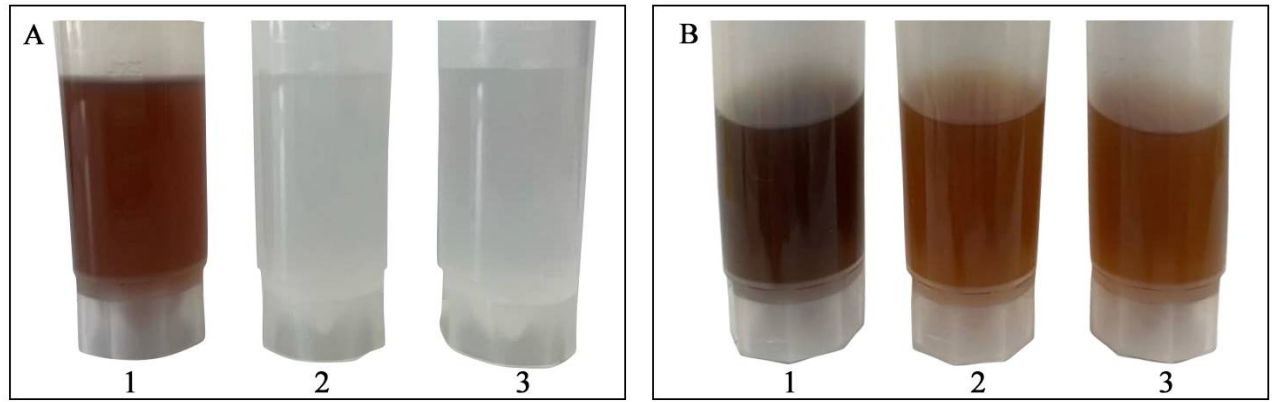

Figure 1. AgNPs biosynthesis. A: cell-free aqueous extract of $S$. cerevisiae $\mathrm{M} 4371$-with $\mathrm{AgNO}_{3}$, 2 - without $\mathrm{AgNO}_{3} ; 3$ - solution of $\mathrm{AgNO}_{3}$.

B: culture fluid supernatant of $S$. cerevisiae $\mathrm{M} 4371$ - with $\mathrm{AgNO}_{3}, 2$ - without $\mathrm{AgNO}_{3}$; 3 - YPD medium with $\mathrm{AgNO}_{3}$

The formation of silver NPs in the solution was confirmed by UV-Vis spectral analysis (Figure 2). As shown in the spectra, the absorption intensity increased with time without shifting the wavelength at which the maximum absorption was observed. This indicates a continuous decrease of the concentration of silver ions and an increase of the concentration of AgNPs, as well as a uniform distribution of nanoparticles in size (Xue et al., 2016; Win et al., 2020).

When a cell-free aqueous yeast extract was used for the biosynthesis of AgNPs (Figure 2, A), a pronounced absorption peak was observed in the wavelength range from 300 to $540 \mathrm{~nm}$ with an average wavelength at which a peak took place at about $425 \mathrm{~nm}$. A similar surface plasmon resonance peak is described for AgNPs smaller than $50 \mathrm{~nm}$ (Win et al., 2020). When the supernatant of S. cerevisiae M437 culture fluid was used for the biosynthesis of AgNPs, an expansion of the absorption spectra was observed (Figure 2, B). This may be due to the aggregation of AgNPs or an increase in their size (Kumari et al., 2020).

Other authors have also shown the possibility of biosynthesis of AgNPs using yeast. When using psychrotrophic yeast Yarrowia lipolytica NCYC 789, the maximum absorption peak was $410 \mathrm{~nm}$ and the average nanoparticle size was $15 \mathrm{~nm}$ (Apte et al., 2013). The absorption peak of AgNPs during their synthesis using extremophilic yeast (genus and species were not indicated by the authors) was $420 \mathrm{~nm}$, and the size was $4-15 \mathrm{~nm}$ (Mourato et al., 2011). While using Saccharomyces cerevisiae for the biosynthesis of AgNPs, obtained nanoparticles with a similar absorption peak, and sizes of 60-110 nm were obtained (Badhusha and Mohideen, 2016). It is considered that the shift of the light absorption peak towards the red spectral range indicates an increase in the size of AgNPs (Win et al., 2020), however, the following experimental data do not always confirm this. Thus, at a peak of 430 $\mathrm{nm}$, the size of AgNPs synthesized using Saccharomyces cerevisiae was 2-20 nm (Korbekandi et al., 2016) and 60-80 nm (Saravanan et al., 2013), at the peak of $440 \mathrm{~nm}-10$ - 
$60 \mathrm{~nm}$ (Sowbarnika et al., 2018), at the peak of $450 \mathrm{~nm}-10 \mathrm{~nm}$ (Roy et al., 2015). Using the yeast Candida sp. VITDKGB, synthesized AgNPs had a size of $87 \mathrm{~nm}$, and the absorption peak was at $430 \mathrm{~nm}$ (Kumar et al., 2011). When using the yeast Rhodotorula sp. ATL72 for the biosynthesis of AgNPs the nanoparticles with a size of 8-21 nm were received, and the light absorption peak was at $450 \mathrm{~nm}$ (Soliman et al., 2018).
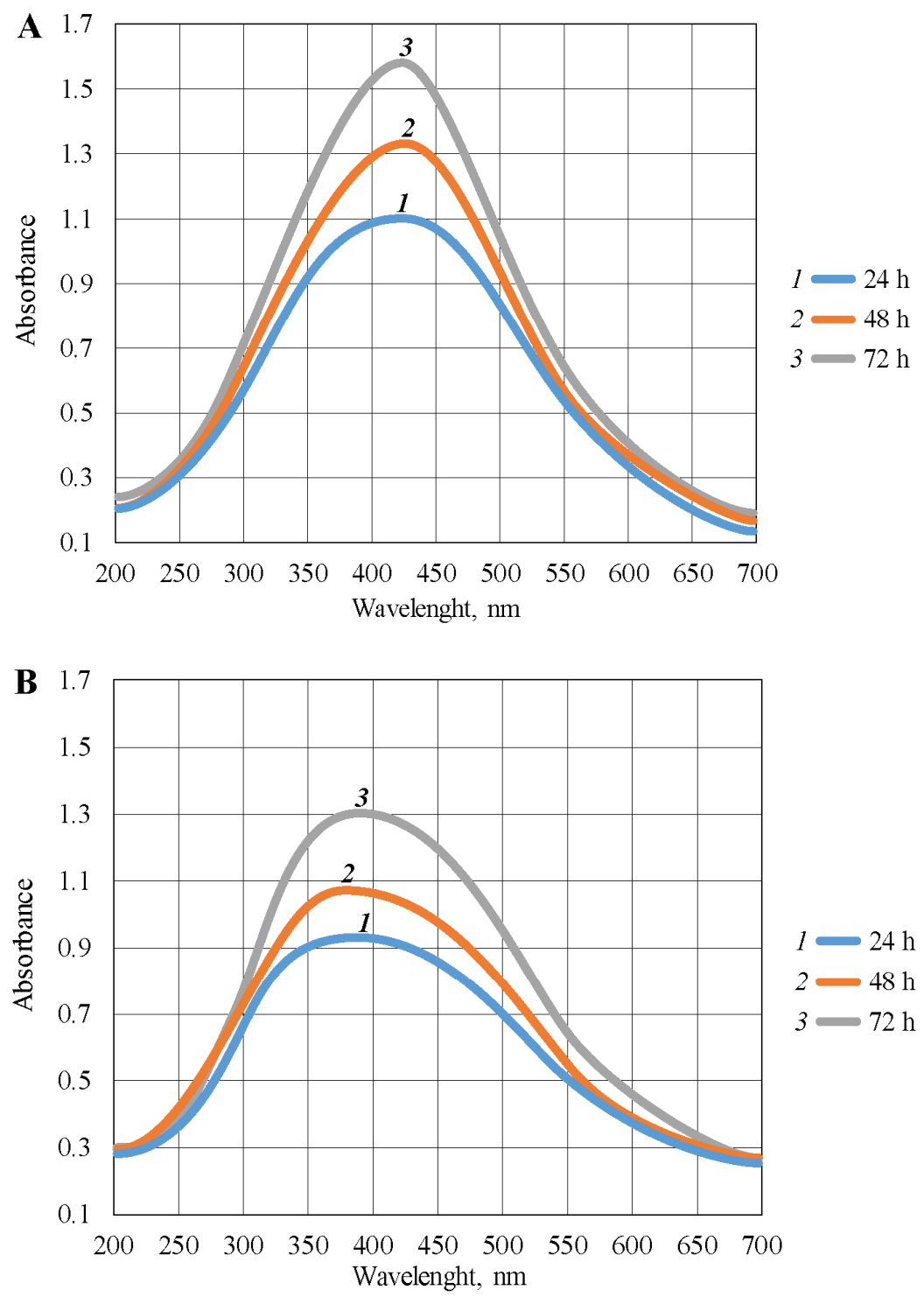

Figure 2. UV-vis spectra of biosynthesized AgNPs using a cell-free yeast extract (A) and culture fluid supernatant (B) 


\section{Size, zeta potential and stability of biosynthesized silver nanoparticles}

The primary results obtained with the DLS are the particle intensity distribution values. The value of the intensity of the distribution of particles in solution is proportional to the value of the square of the molecular weight. This distribution is used to determine the increase in particle size in the sample. To analyze the reliability of the formation of nanocomplexes, we compared the PdI (polydispersity index) and Z-Average (average semi-variant) indices. $\mathrm{Z}$-average is the main and most stable parameter of this method. It defines the average particle size as "the average value of the harmonic average particle diameter". The polydispersity index can determine the distribution of particles by size or mass. PdI is defined as the ratio of weight average molecular weight to number average molecular weight. This is a dimensionless value of the width of the particle size distribution. The calculations used for the determination of size and PDI parameters are defined in the ISO standard documents 13321:1996 E and ISO 22412:2008 (Worldwide, 2011). PDI value which is higher than 0,7 indicates that the sample has a very wide particle size distribution and is probably not suitable to be analysed by the dynamic light scattering (DLS) technique (Danaei et al., 2018). The higher the PdI value, the less monodisperse nanoparticles are in solution. Based on this, the lower the PdI value, the less capable of aggregation of nanoparticles in the sample and their size is in a narrow range of values (Skora et al., 2021).

The isolated nanoparticles were dispersed in double-distilled water at neutral $\mathrm{pH}$ and studied using Zetasizer Nano ZS. The size of AgNPs, which was synthesized using the supernatant of the culture medium of $S$. cerevisiae M437, was determined. The major part of nanoparticles has a size of $152.2 \mathrm{~nm}$, which is close to the value of Z-average (Table 1). When testing the correctness of the null hypothesis using the Wilcoxon test, it was found that the values of the size distribution in Peak 1 and Z-average differ significantly $(p<0.05)$. The value of $\mathrm{PdI}$ is 0.3 , which corresponds to a rather narrow range of particle size distribution.

Table 1

Characteristics of synthesized AgNPs using supernatant of culture medium S. cerevisiae M437

\begin{tabular}{|c|c|c|c|}
\hline \multirow{2}{*}{ Index } & \multicolumn{3}{|c|}{ Measurements were performed immediately after biosynthesis } \\
\hline & Peak 1 & Peak 2 & Peak 3 \\
\hline Size, nm & $152.2[146.1 ; 154.2]$ & $4848.0[4519.0 ; 4997.0]$ & $0.0[0.0 ; 13.1]$ \\
\hline Volume, \% & $94.4[91.2 ; 96.2]$ & $5.1[3.9 ; 7.2]$ & $0.0[0.0 ; 1.9]$ \\
\hline Z-average, $\mathrm{nm}$ & \multicolumn{3}{|c|}{$129.6[127.5 ; 132.0]$} \\
\hline \multirow[t]{3}{*}{ PdI } & \multicolumn{3}{|c|}{$0.3[0.3 ; 0.4]$} \\
\hline & \multicolumn{3}{|c|}{ Measurements were performed after 45 days of storage at $4^{\circ} \mathrm{C}$} \\
\hline & Peak 1 & Peak 2 & Peak 3 \\
\hline Size, nm & $204.4[176.5 ; 229.1]$ & $3779.0[2448.5 ; 4504.0]$ & $0.0[0.0 ; 2148.0]$ \\
\hline Volume, $\%$ & $91.2[80.7 ; 91.9]$ & $8.8[8.2 ; 15.6]$ & $0.0[0.0 ; 3.8]$ \\
\hline Z-average, $\mathrm{nm}$ & \multicolumn{3}{|c|}{$156.1[153.3 ; 163.8]$} \\
\hline PdI & \multicolumn{3}{|c|}{$0.5[0.5 ; 0.6]$} \\
\hline
\end{tabular}

In addition, the stability of dispersed in double-distilled water nanoparticles after 45 days storage at $4{ }^{\circ} \mathrm{C}$ was evaluated (Table 1). After storage, some changes in AgNPs size were observed. When testing the correctness of the null hypothesis using the Wilcoxon test, 
it was found that the size distribution in Peak 1 and Z-average value was not differing significantly $(p>0.05)$. This indicates that after storage at these conditions, AgNPs have a spherical shape. The PdI value was 0.5 , which corresponds to the average range of the nanoparticle size distribution range. Therefore, AgNPs after storage were likely to be degraded, or there were continuous transformation processes in the suspension, which led to an increase in the range of hydrodynamic diameter values. It should be noted that after saving the volume distribution changed a little (Table 1) and these changes were insignificant $(p>0.05)$. However, there was a significant $(p<0.01)$ increase in PdI by $40 \%$, which directly indicated the increase in the range of hydrodynamic diameter values. This in turn confirms the presence of disintegration reactions and aggregation of the formed nanoparticles.

After storage, the hydrodynamic diameter of the synthesized AgNPs (Peak 1, Figure 3) increases by $25.5 \%(p<0.05)$ and the Z-Average value increases by $17 \%(p<0.01)$.

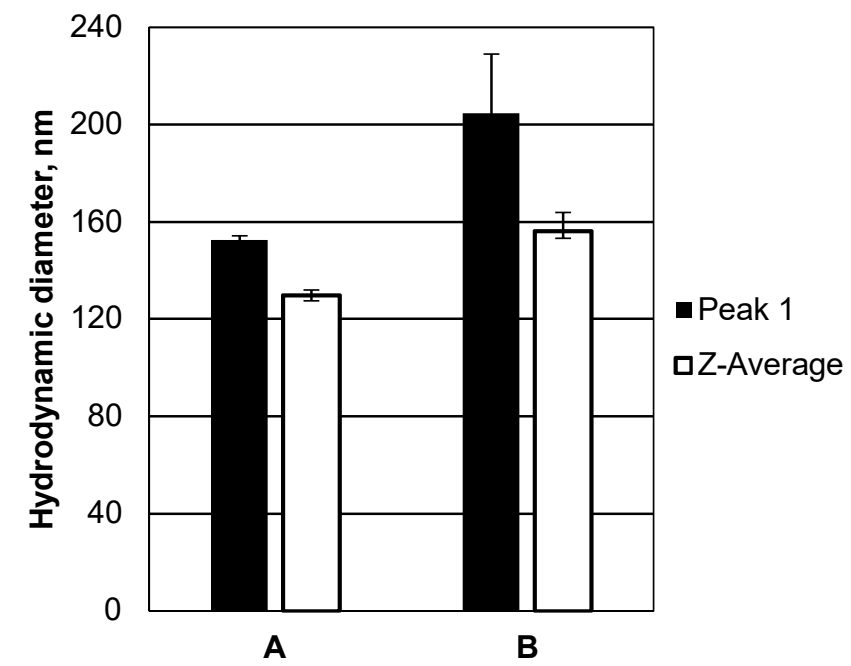

Figure 3. Sizes of only synthesized (A) AgNPs using S. cerevisiae M437 supernatant and after storage for 45 days (B)

Characteristics of AgNPs that were obtained using cell-free aqueous extract of S. cerevisiae M437 are given in table 2. The value of the distribution of nanoparticles in size in Peak 1 and Z-Average do not differ significantly $(p>0.05)$. The PdI value corresponds to a narrow range of particle size distribution. After storage of AgNPs for 45 days, there were some changes in the size and distribution of nanoparticles (Table 2). The value of PdI after storage increases by $25 \%$, but this increase is not significant $(p>0.05)$. This suggests that AgNPs remain stable in colloidal solution.

When comparing the sizes of AgNPs obtained using a cell-free aqueous extract of S. cerevisiae M437 before and after storage (Peak 1, Figure 4), we see that the change in their hydrodynamic diameter is statistically insignificant $(p>0.05)$. The Z-Average decreases by $7 \%(p>0.05)$, which is also unreliable. That is, there are no changes in the shape of the nanoparticles. 
Table 2

Size distribution of AgNPs obtained using cell-free aqueous extract of S. cerevisiae M437

\begin{tabular}{|l|c|c|c|}
\hline \multirow{2}{*}{ Index } & Measurements were performed immediately after biosynthesis \\
\cline { 2 - 4 } & Peak 1 & Peak 2 & Peak 3 \\
\hline Size, $\mathrm{nm}$ & $163.4[144.2 ; 186.1]$ & $0.0[0.0 ; 4444.5]$ & $0.0[0.0 ; 0.0]$ \\
\hline Volume, \% & $100.0[97.8 ; 100.0]$ & $0.0[0.0 ; 2.2]$ & $0.0[0.0 ; 0.0]$ \\
\hline Z-Average, $\mathrm{nm}$ & \multicolumn{3}{|c|}{$143.6[138.4 ; 164.1]$} \\
\hline PdI & \multicolumn{3}{|c|}{$0.3[0.3 ; 0.4]$} \\
\hline & Measurements were performed after 45 days of storage at 4 ${ }^{\circ} \mathbf{C}$ \\
\hline & Peak 1 & Peak 2 & Peak 3 \\
\hline Size, nm & $175.7[173.7 ; 183.9]$ & $4561.0[343.0 ; 4714,0]$ & $0.0[0.0 ; 2485.5]$ \\
\hline Volume, \% & $95.2[90.8 ; 96.4]$ & $4.8[3.6 ; 6.1]$ & $0.0[0.0 ; 1.6]$ \\
\hline Z-Average, $\mathrm{HM}$ & \multicolumn{3}{|c}{$133.3[132.0 ; 133.9]$} \\
\hline PdI & \multicolumn{3}{|c|}{$0.4[0.4 ; 0.4]$} \\
\hline
\end{tabular}

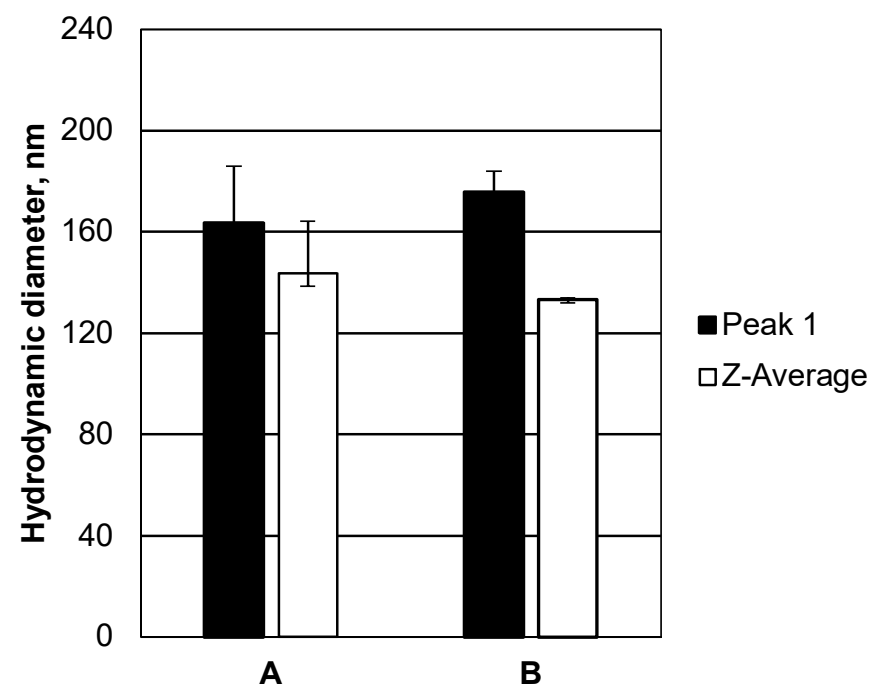

Figure 4. Sizes of synthesized (A) AgNPs using a cell-free aqueous yeast extract and after storage for 45 days (B)

Comparing the sizes of biogenic AgNPs obtained using the above two methods, it was evident that the value of their hydrodynamic diameter did not differ statistically (Figure 5). The difference between the Z-average values was significant $(p<0.01)$. This suggests that the shape of AgNPs obtained by different methods was different, which was confirmed by the TEM data. 


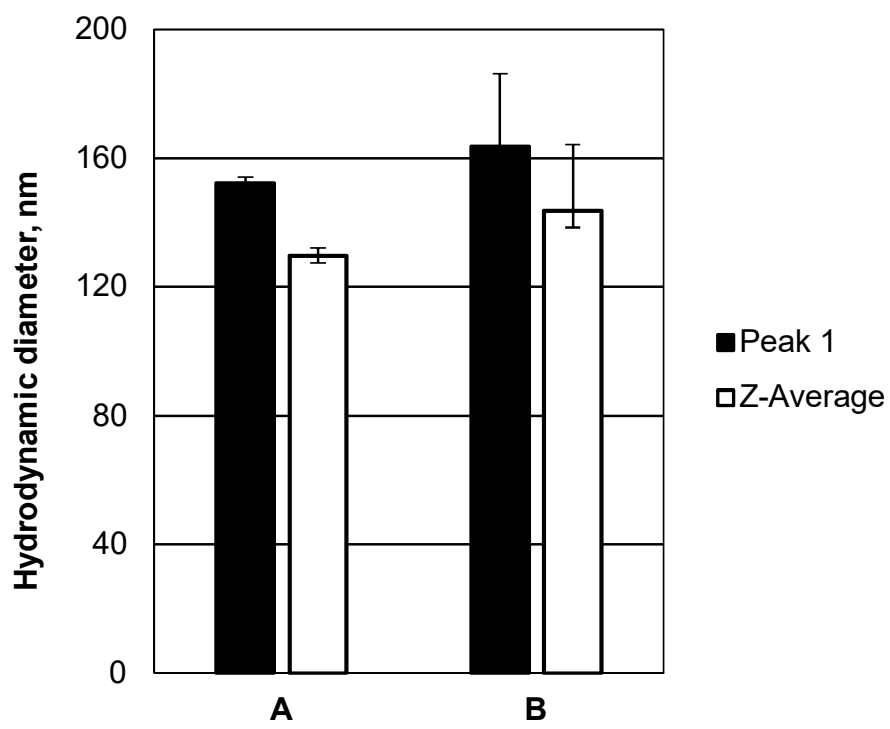

Figure 5. Hydrodynamic diameter of AgNPs obtained using supernatant (A) and cell-free aqueous extract (B) S. cerevisiae M437

We also compared the characteristics of AgNPs obtained using cell-free aqueous extract or supernatant of S. cerevisiae M437 after storage for 45 days at $4{ }^{\circ} \mathrm{C}$ (Figure 6). The hydrodynamic diameter of nanoparticles and the Z-average value were not statistically different $(p>0.05)$.

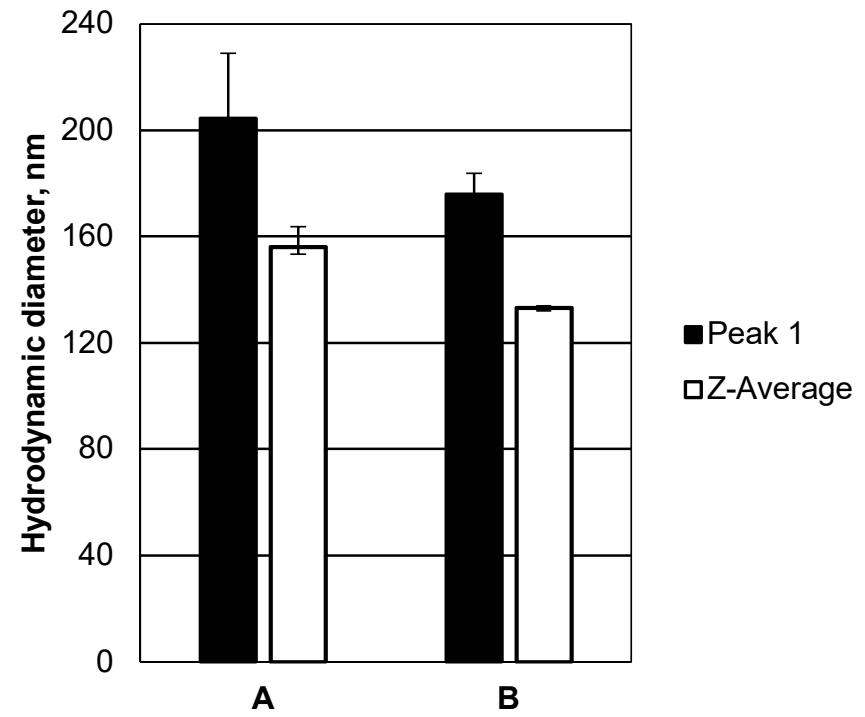

Figure 6. Hydrodynamic diameter of AgNPs obtained using supernatant (A) and cell-free aqueous extract (B) from $S$. cerevisiae M437 after storage for 45 days 
Comparing the polydispersity of the studied AgNPs shows that the nanoparticles obtained using a cell-free aqueous extract from S. cerevisiae M437 are 27.2\% $(p<0.01)$ less polydisperse than the nanoparticles obtained using the supernatant of the S. cerevisiae M437 culture liquid (Tables 1 and 2). This indicates a more uniform distribution of nanoparticles in size after storage under the specified conditions.

Zeta potential is an important indicator of the surface charge of nanoparticles (Kthiri et al., 2021). The surface charge of AgNPs is related to their dispersity and stability (Leo et al., 2013). The high value of the negative zeta potential indicates long-term stability and dispersion, lack of flocculation and the tendency to form agglomerates of biogenic AgNPs due to the repulsion of negatively charged particles (Skoglund et al., 2017; Win et al., 2020; Foujdar et al., 2021).

The value of the zeta potential for AgNPs obtained using the supernatant of the culture liquid of S. cerevisiae M437 was $-13.6[-13.8 ;-13.1] \mathrm{mV}$. After 45 days of storage at $4{ }^{\circ} \mathrm{C}$, the zeta potential increased by $11.7 \%$ and was $-12.0[-12.6 ;-11.0] \mathrm{mV}$. For AgNPs obtained using the cell-free aqueous extract from $S$. cerevisiae M437, the value of the zeta potential was $-13.7[-14.5 ;-13.5] \mathrm{mV}$, and after storage it was $-19.3[-20.1 ;-18.6] \mathrm{mV}$. That is a $29 \%$ decrease in the zeta potential.

Obtained results indicate that negatively charged ions are present on the surface of AgNPs. Due to the repulsion between them, biogenic AgNPs were stable in solution for 45 days of storage. At the same time, nanoparticles obtained using a cell-free aqueous extract from S. cerevisiae M437 were more stable due to the more negative charge of their surface.

\section{Electron microscopy}

The morphology and size of biogenic AgNPs were examined by transmission electron microscopy (TEM). Silver nanoparticles were relatively homogeneous and close to a spherical shape (Figure 7) with dimensions of less than $30 \mathrm{~nm}$ and tended to form groups or aggregates.

The median size of nanoparticles and their aggregates was calculated according to the internal scale mark (bar) indicated on the microphotographs (Table 3).

\section{Size of biogenic silver nanoparticles}

Table 3

\begin{tabular}{|c|c|c|c|c|}
\hline \multirow{2}{*}{$\begin{array}{c}\text { Origin of the } \\
\text { nanoparticles }\end{array}$} & \multicolumn{2}{|c|}{ Aggregate } & \multicolumn{2}{c|}{ Separate nanoparticle } \\
\cline { 2 - 5 } & Width, $\mathbf{n m}$ & Length, $\mathbf{n m}$ & Width, $\mathbf{n m}$ & Length, nm \\
\hline $\begin{array}{c}\text { Obtained using a cell- } \\
\text { free aqueous extract } \\
\text { from S. cerevisiae M437 }\end{array}$ & 39.1 & 32.0 & 21.3 & 14.2 \\
\hline $\begin{array}{c}\text { Obtained using the } \\
\text { supernatant of the } \\
\text { culture liquid of }\end{array}$ & $23.1 ; 69.2]$ & {$[23.1 ; 47.9]$} & {$[14.2 ; 24.9]$} & {$[14.2 ; 21.3]$} \\
S. cerevisiae M437 & {$[132.0 ; 257.4]$} & 109.2 & 15.6 & 15.6 \\
{$[60.6 ; 195.0]$} & {$[10.8 ; 32.4]$} & {$[10.8 ; 27.0]$} \\
\hline
\end{tabular}



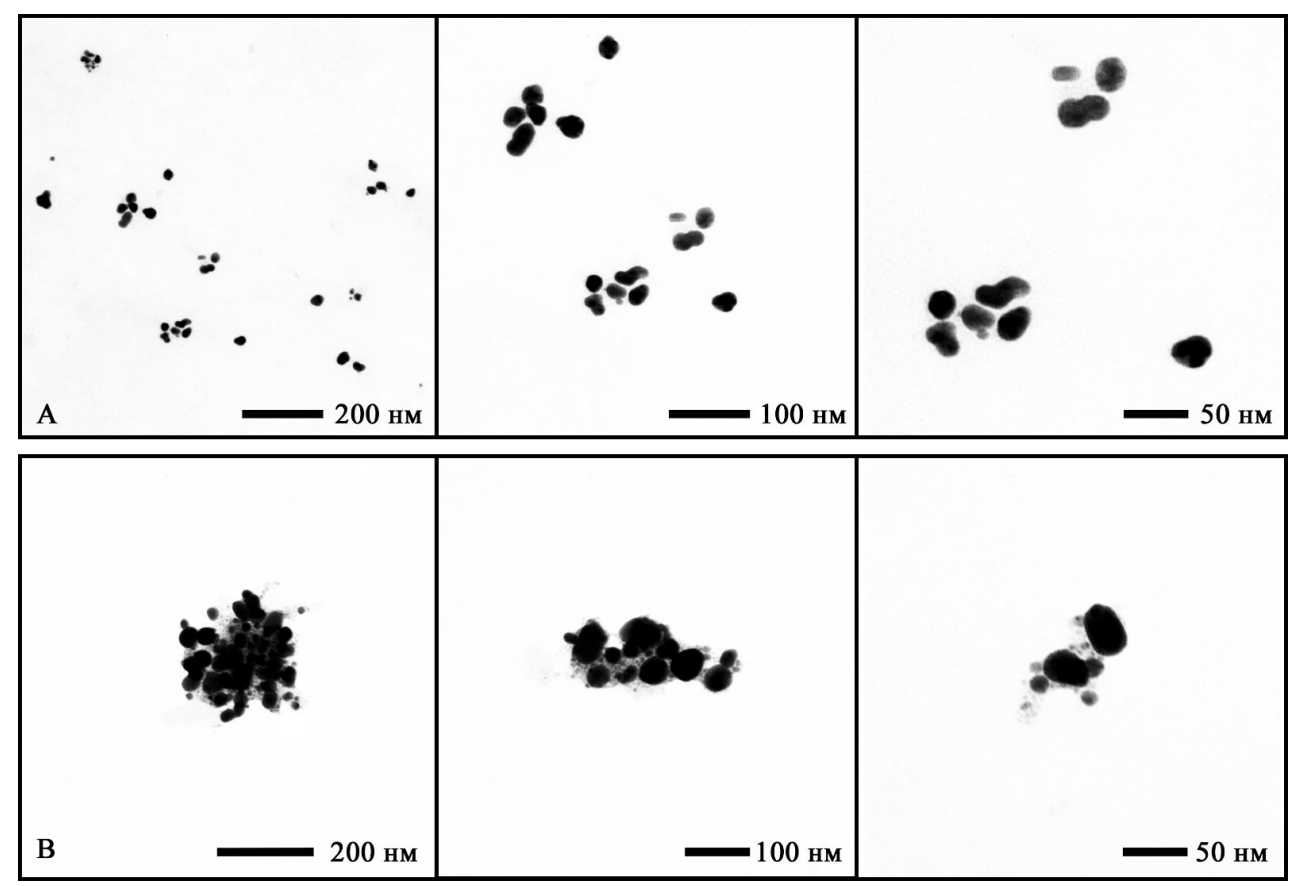

Figure 7. Electronic micrographs of AgNPs obtained using cell-free aqueous extract (A) and supernatant of culture liquid S. cerevisiae M437 (B)

There are no significant differences in size between AgNPs synthesized using the supernatant and using a cell-free aqueous extract of the studied yeast (Table 3). During the synthesis of AgNPs using a cell-free extract, there is a tendency to the formation of nanoparticles close to oval, and during the synthesis of AgNPs using the supernatant, they are close to spherical. It is also noticeable the feature of AgNPs synthesized using the supernatant $S$. cerevisiae M437 to form relatively large aggregates of nanoparticles.

It was previously reported that when using the supernatant of the culture liquid Candida utilis NCIM 3469, no biosynthesis of AgNPs was observed when $\mathrm{AgNO}_{3}$ was added to a final concentration of $2 \mathrm{mM}$ at a temperature of $30^{\circ} \mathrm{C}$ (Waghmare et al., 2015). At the same time, using the supernatant of Candida glabrata at room temperature of biosynthesis, it was possible to obtain AgNPs of spherical and oval shapes with sizes of 2-15 nm (Jalal et al., 2018). An interesting study is where $S$. cerevisiae BY4741 was grown under the influence of a moderate static magnetic field, after which the culture broth supernatant was used for the biosynthesis of AgNPs. The resulting nanoparticles were mostly spherical and had a size of 2-12 nm (Kthiri et al., 2021).

Using a cell-free extract of red yeast Phaffia rhodozyma, quasi-spherical AgNPs with a diameter of $4.1 \mathrm{~nm}$ were obtained. The silver nitrate was added to the reaction mixture at a final concentration of 0.1 M (Ronavari et al., 2018). Synthesis of AgNPs using cell-free aqueous extract of $S$. cerevisiae was shown by Skora with co-authors. The authors obtained spherical AgNPs with an average diameter of $20.1 \mathrm{~nm}$. The temperature of biosynthesis was $60{ }^{\circ} \mathrm{C}$ (Skora et al., 2021). 
Thus, depending on the choice of conditions for biogenic synthesis of nanoparticles, it is possible to obtain different sizes of AgNPs. The sizes of AgNPs, which were determined using TEM, were smaller than sizes determined using the Zetasizer Nano ZS. This can be explained by the presence of surface proteins, carbohydrates, and other cellular compounds that can participate in the stabilization of biogenic nanoparticles, which were measured by Zetasizer. However, these compounds are not fixed when using the TEM method, since they cannot be retained on the surface of nanoparticles in vacuum under an electron beam (Xue et al., 2016). Also, it should be said that the Zetasizer Nano device cannot differentiate individual nanoparticles from their aggregates, therefore it perceives a separate aggregate as a separate particle.

The obtained different values of the sizes of biogenic AgNPs can be explained by the constantly running processes of decomposition and formation of new aggregates of nanoparticles occurring simultaneously. This hypothesis can be refined in future studies using stabilizers and the study of samples using the Zetasizer Nano in dynamics for a long time.

\section{Conclusions}

The possibility of mediated synthesis of AgNPs by the Saccharomyces cerevisiae M437 yeast strain from the collection of live cultures of the Department of Biotechnology and Microbiology of the National University of Food Technologies have been shown. In this case, the synthesis of AgNPs was observed both when using the culture fluid supernatant and the cell-free aqueous extract from S. cerevisiae M437. The size and shape of the nanoparticles that were obtained using these two methods did not actually differ. But we found a difference in the specific sizes of AgNPs using different methods (Zetasizer Nano ZS and TEM). Perhaps this difference is due to organic compounds involved in the formation and stabilization of nanoparticles. However, these compounds are not fixed when using the TEM method, since they cannot be retained on the surface of nanoparticles in vacuum under an electron beam. A difference in the stability of biogenic AgNPs after storage in solution for 45 days was also observed. At the same time, the nanoparticles obtained with the use of a cell-free aqueous yeast extract turned out to be more stable. In the future, the antimicrobial activity of biogenic AgNPs with a view to their further use in the food industry will be studied.

\section{References}

Amer S.A., Abushady H.M., Refay R.M., Mailam M.A. (2021), Enhancement of the antibacterial potential of plantaricin by incorporation into silver nanoparticles, J. Genet. Eng. Biotechnol., 19(13), pp. 1-16, DOI: 10.1186/s43141-020-00093-z.

Apte M., Sambre D., Gaikawad S., Joshi S., Bankar A., Kumar A.R., Zinjarde S. (2013) Psychrotrophic yeast Yarrowia lipolytica NCYC 789 mediates the synthesis of antimicrobial silver nanoparticles via cell-associated melanin, AMB Expr, 3, 32, DOI: 10.1186/2191-08553-32.

Badhusha M.S.M., Mohideen M.M.A.K. (2016), Biosynthesis of silver nanoparticles using Saccharomyces cerevisiae with different $\mathrm{pH}$ and study of antimicrobial activity against bacterial pathogens, Chemical Science Transactions, 5(4), pp. 906-911, DOI:10.7598/cst2016.1275. 
Bahrulolum H., Nooraei S., Javanshir N., Tarrahimofrad H., Mirbagheri V.S., Easton A.J., Ahmadian G. (2021), Green synthesis of metal nanoparticles using microorganisms and their application in the agrifood sector, J. Nanobiotechnol., 19, 86, DOI: 10.1186/s12951-02100834-3.

Bocate K.P., Reis G.F., Souza P.C., Admilton G., Junior O., Durán N., Nakazato G., Furlaneto M.C., Almeida R.S., Panagio L.A. (2019), Antifungal activity of silver nanoparticles and simvastatin against toxigenic species of Aspergillus, International Journal of Food Microbiology, 291, pp. 79-86, DOI: 10.1016/j.ijfoodmicro.2018.11.012.

Castillo-Henríquez L., Alfaro-Aguilar K., Ugalde-Álvarez J., Vega-Fernández L., Oca-Vásquez G.M., Vega-Baudrit J.R. (2020), Green synthesis of gold and silver nanoparticles from plant extracts and their possible applications as antimicrobial agents in the agricultural area, Nanomaterials, 10, 1763, DOI: 10.3390/nano10091763.

Chandhru M., Logesh R., Rani S.K., Ahmed N., Vasimalai N. (2019), One-pot green route synthesis of silver nanoparticles from jack fruit seeds and their antibacterial activities with escherichia coli and salmonella bacteria, Biocatal. Agric. Biotechnol., 20, 101241, DOI: 10.1016/j.bcab.2019.101241.

Danaei M., Dehghankhold M., Ataei S., Hasanzadeh Davarani F., Javanmard R., Dokhani A., Khorasani S., Mozafari M.R. (2018), Impact of particle size and polydispersity index on the clinical applications of lipidic nanocarrier systems, Pharmaceutics, 10(2), 57, DOI: 10.3390/pharmaceutics10020057.

Du J., Hu Z., Yu Z., Li H., Pan J., Zhao D., Bai Y. (2019), Antibacterial activity of a novel Forsythia suspensa fruit mediated green silver nanoparticles against food-borne pathogens and mechanisms investigation, Mater. Sci. Eng. C, 102, pp. 247-253, DOI: 10.1016/j.msec.2019.04.031.

Ernest V., Shiny P.J., Mukherjee A., Chandrasekaran N. (2012), Silver nanoparticles: a potential nanocatalyst for the rapid degradation of starch hydrolysis by $\alpha$-amylase, Carbohydrate Research, 352, pp. 60-64, DOI: 10.1016/j.carres.2012.02.009.

Fay M.P., Malinovsky Y. (2017), Confidence intervals of the Mann-Whitney parameter that are compatible with the Wilcoxon-Mann-Whitney test., Statistics in Medicine. 37(27), pp. 3991-4006, DOI: 10.1002/sim.7890.

Foujdar R., Chopra H.K., Bera M.B., Chauhan A.K., Mahajan P. (2021). Effect of probe ultrasonication, microwave and sunlight on biosynthesis, bioactivity and structural morphology of Punica granatum peel's polyphenols-based silver nanoconjugates, Waste Biomass Valor, 12, pp. 2283-2302, DOI: 10.1007/s12649-020-01175-2.

Gasparyana V.K., Bazukyan I.L. (2013), Lectin sensitized anisotropic silver nanoparticles for detection of some bacteria, Analytica Chimica Acta, 766, pp. 83-87. DOI: 10.1016/j.aca.2012.12.015.

Pal G., Rai P., Pandey A. (2019). Chapter 1 - Green synthesis of nanoparticles: A greener approach for a cleaner future, In Micro and Nano Technologies, Green Synthesis, Characterization and Applications of Nanoparticles, Elsevier, pp. 1-26, DOI: 10.1016/B978-0-08-102579-6.00001-0.

Grasso G., Zane D., Dragone R. (2020), Microbial nanotechnology: challenges and prospects for green biocatalytic synthesis of nanoscale materials for sensoristic and biomedical applications, Nanomaterials (Basel), 10, 11, DOI: 10.3390/nano10010011.

Halder S., Ahmed A.N., Gafur M.A., Seong G., Hossain M.Z. (2021), Size-controlled facile synthesis of silver nanoparticles by chemical reduction method and analysis of their antibacterial performance, ChemistrySelect, 6(36), pp. 9714-9720, DOI: 10.1002/slct.202101362.

Hashim N., Paramasivam M., Tan J.S., Kernain D., Hussin M.H., Brosse N., Gambier F., Raja P.B. (2020), Green mode synthesis of silver nanoparticles using Vitis vinifera's tannin and 
screening its antimicrobial activity/apoptotic potential versus cancer cells, Materials Today Communications, 25, 101511, DOI: 10.1016/j.mtcomm.2020.101511.

Hovhannisyana V.A., Bazukyanb I.L., Gasparyan V.K. (2017), Application of silver nanoparticles and CdSe quantum dots sensitized with of C-like lectin for detection of St. aureus. Comparison of various approaches, Talanta, 175, pp. 366-369, DOI: 10.1016/j.talanta.2017.07.062.

Huang H., Shan K., Liu J., Tao X., Periyasamy S., Durairaj S., Jiang Z., Jacob J.A. (2020), Synthesis, optimization and characterization of silver nanoparticles using the catkin extract of Piper longum for bactericidal effect against food-borne pathogens via conventional and mathematical approaches, Bioorganic Chemistry, 103, 104230, DOI: 10.1016/j.bioorg.2020.104230.

Jalal M., Ansari M.A., Alzohairy M.A., Ali S.G., Khan H.M. (2018), Biosynthesis of silver nanoparticles from oropharyngeal Candida glabrata isolates and their antimicrobial activity against clinical strains of bacteria and fungi, Nanomaterials, 8(8), 586, DOI: 10.3390/nano8080586.

Kandasamy S., Xiaowen Hu, Ramachandran C., Deog-Hwan Oh, Kandasamy K., Myeong-Hyeon W. (2020), Biogenic silver nanoparticles-polyvinylpyrrolidone based glycerosomes coating to expand the shelf life of fresh-cut bell pepper (Capsicum annuum L. var. grossum (L.) Sendt), Postharvest Biology and Technology, 160, 111039, DOI: 10.1016/j.postharvbio.2019.111039.

Korbekandi H., Mohseni S., Jouneghani R.M., Pourhossein M., Iravani S. (2016), Biosynthesis of silver nanoparticles using Saccharomyces cerevisiae, Artif. Cells, Nanomed., Biotechnol., 44(1), pp. 235-239, DOI: 10.3109/21691401.2014.937870.

Krishnakumar S., Janani P., Mugilarasi S., Kumari G., Janney J.B. (2018), Chemical induced fabrication of silver nanoparticles (Ag-NPs) as nanocatalyst with alpha amylase enzyme for enhanced breakdown of starch, Biocatalysis and Agricultural Biotechnology, 15, pp. 377 383, DOI: 10.1016/j.bcab.2018.06.016.

Kthiri A., Hamimed S., Othmani A., Landoulsi A., O’Sullivan S., Sheehan D. (2021), Novel static magnetic field effects on green chemistry biosynthesis of silver nanoparticles in Saccharomyces cerevisiae, Sci Rep, 11, 20078, DOI: 10.1038/s41598-021-99487-3.

Kumar D., Karthik L., Kumar G., Roa K.B. (2011), Biosynthesis of silver anoparticles from marine yeast and their antimicrobial activity against multidrug resistant pathogens, Pharmacologyonline, 2011, 3, pp. 1100-1111.

Kumar J.A., Krithiga T., Manigandan S., Sathish S., Renita A.A., Prakash P., Prasad B.S.N., Kumar T.R.P., Rajasimman M., Hosseini-Bandegharaei A., Prabu D., Crispin S. (2021), A focus to green synthesis of metal/metal based oxide nanoparticles: Various mechanisms and applications towards ecological approach, Journal of Cleaner Production, 324, 129198, DOI: 10.1016/j.jclepro.2021.129198.

Kumari R.M., Kumar V., Kumar M., Pareek N., Nimesh S. (2020), Assessment of antibacterial and anticancer capability of silver nanoparticles extracellularly biosynthesized using Aspergillus terreus, Nano Express, 1(3), 030011, DOI: 10.1088/2632-959X/abc2e4.

Kuuliala L., Pippuri T., Hultman J., Auvinen S.-M., Kolppo K., Nieminen T., Karp M., Bjorkroth J., Kuusipalo J., Jaaskelainen E. (2015), Preparation and antimicrobial characterization of silver-containing packaging materials for meat, Food Packaging and Shelf Life, 6, pp. 5360, DOI: 10.1016/j.fps1.2015.09.004.

Lekha D.C., Shanmugam R., Madhuri K., Dwarampudi L.P., Bhaskaran M., Kongara D., Tesfaye J.L., Nagaprasad N., Bhargavi V.L.N., Krishnaraj R. (2021), Review on silver nanoparticle synthesis method, antibacterial activity, drug delivery vehicles, and toxicity pathways: recent advances and future aspects, Journal of Nanomaterials, 2021, 4401829, DOI: $10.1155 / 2021 / 4401829$. 
Leo B.F., Chen S., Kyo Y., Herpoldt K., Terrill N.J., Dunlop I.E., McPhail D.S., Shaffer M.S., Schwander S., Gow A., Zhang J., Chung K.F., Tetley T.D., Porter A.E., Ryan M.P. (2013), The stability of silver nanoparticles in a model of pulmonary surfactant, Environ Sci Technol, 47, pp. 11232-11240, DOI: 10.1021/es403377p.

Liu J., Zheng D., Zhong L., Gong A., Wu S., Xie Z. (2021), Biosynthesis of biocompatibility $\mathrm{Ag}_{2} \mathrm{Se}$ quantum dots in Saccharomyces cerevisiae and its application, Biochemical and Biophysical Research Communications, 544, pp. 60-64, DOI: 10.1016/j.bbrc.2021.01.071.

Loira I., Morata A., Escott C., Del Fresno J.M., Tesfaye W., Palomero F., Suárez-Lepe J.A. (2020), Applications of nanotechnology in the winemaking process, Eur Food Res Technol, 246, pp. 1533-1541, DOI: 10.1007/s00217-020-03519-7.

Ma X., Lin X., Xu X., Wang Z. (2021), Fabrication of gold/silver nanodimer SERS probes for the simultaneous detection of Salmonella typhimurium and Staphylococcus aureus, Microchim Acta, 188, 202, DOI: 10.1007/s00604-021-04791-4.

Mourato A., Gadanho M., Lino A.R., Tenreiro R. (2011), Biosynthesis of crystalline silver and gold nanoparticles by extremophilic yeasts, Bioinorganic Chemistry and Applications, 2011, 546074, DOI: 10.1155/2011/546074.

Oliveira S.S., Braga G.C., Cordeiro N.K., Stangarlin J.R., Alves H.J. (2021), Green synthesis of silver nanoparticles with Euphorbia tirucalli extract and its protection against microbial decay of strawberries during storage, J. Food Sci. Technol., DOI: 10.1007/s13197-02105217-y.

Paidari S., Ahari H. (2021), The effects of nanosilver and nanoclay nanocomposites on shrimp (Penaeus semisulcatus) samples inoculated to food pathogens, Food Measure, 15, pp. 31953206, DOI: 10.1007/s11694-021-00905-X.

Qiao Z., Cai Q., Fu Y., Lei C., Wenge Y. (2021), Visual and quantitative detection of E. coli O157:H7 by coupling immunomagnetic separation and quantum dot-based paper strip., Anal Bioanal Chem, 413, pp. 4417-4426, DOI: 10.1007/s00216-021-03395-4.

Reddy N.V., Li H., Hou T., Bethu M.S., Ren Z., Zhang Z. (2021), Phytosynthesis of silver nanoparticles using Perilla frutescens leaf extract: characterization and evaluation of antibacterial, antioxidant, and anticancer activities, International journal of nanomedicine, 16, pp. 15-29, DOI: 10.2147/IJN.S265003.

Ronavari A., Igaz N., Gopisetty M. K., Szerencses B., Kovacs D., Papp C., Vagvölgyi C., Boros I.M., Konya Z., Kiricsi M., Pfeiffer I. (2018), Biosynthesized silver and gold nanoparticles are potent antimycotics against opportunistic pathogenic yeasts and dermatophytes, International journal of nanomedicine, 13, pp. 695-703, DOI: 10.2147/IJN.S152010.

Rosman N.S.R., Harun N.A., Idris I., Ismail W.I.W. (2020), Eco-friendly silver nanoparticles (AgNPs) fabricated by green synthesis using the crude extract of marine polychaete, Marphysa moribidii: biosynthesis, characterisation, and antibacterial applications, Heliyon, 6(11), e05462, DOI: 10.1016/j.heliyon.2020.e05462.

Roy K., Sarkar C.K., Ghosh, C.K. (2015), Photocatalytic activity of biogenic silver nanoparticles synthesized using yeast (Saccharomyces cerevisiae) extract, Appl Nanosci, 5, pp. 953-959, DOI: $10.1007 / \mathrm{s} 13204-014-0392-4$.

Sachdev D., Joshi A., Taneja N.K., Pasricha R. (2021) Chapter 7 - Emerging silver nanomaterials for smart food packaging in combating food-borne pathogens, In Nanobiotechnology for Plant Protection, Silver Nanomaterials for Agri-Food Applications, Elsevier, pp. 147-185, DOI: 10.1016/B978-0-12-823528-7.00009-3.

Gil-Sanchez I., Monge M., Miralles B., Armentia G., Cueva C., Crespo J., Luzuriaga J.M.L., Olmos M.E., Bartolomé B., Llano D.G., Moreno-Arribas M.V., (2019), Some new findings on the potential use of biocompatible silver nanoparticles in winemaking, Innovative Food Science \& Emerging Technologies, 51, pp. 64-72, DOI: 10.1016/j.ifset.2018.04.017.

Saravanan M., Amelash T., Negash L. Gebreyesus A., Selvaraj A., Rayar V., Dheekonda K. (2013), Extracellular biosynthesis and biomedical application of silver nanoparticles 
synthesized from baker's yeast, International Journal of Research in Pharmaceutical and Biomedical Sciences, 4(3), pp. 822-828.

Shahat M.S., Ibrahim M.I., Osheba A.S., Taha I.M. (2020), Preparation and characterization of silver nanoparticles and their use for improving the quality of apricot fruits, Al-Azhar Journal of Agricultural Research, 45(1), pp. 38-55, DOI: 10.21608/ajar.2020.126625.

Silvan J.M., Zorraquin-Pena I., Gonzalez de Llano D., Moreno-Arribas M.V., MartinezRodriguez A.J. (2018), Antibacterial activity of glutathione-stabilized silver nanoparticles against Campylobacter multidrug-resistant strains, Front. Microbiol., 9, 458, DOI: 10.3389/fmicb.2018.00458.

Skoglund S., Blomberg E., Wallinder I.O., Grillo I., Pedersen J.S., Bergström L.M. (2017) A novel explanation for the enhanced colloidal stability of silver nanoparticles in the presence of an oppositely charged surfactant, Phys Chem Chem Phys, 19, pp. 28037-28043, DOI: 10.1039/C7CP04662F.

Skora B., Krajewska U., Nowak A., Dziedzic A., Barylyak A., Kus-Liskiewicz M. (2021), Noncytotoxic silver nanoparticles as a new antimicrobial strategy, Sci. Rep., 11, 13451, DOI: 10.1038/s41598-021-92812-w.

Soliman H., Elsayed A., Dyaa A. (2018), Antimicrobial activity of silver nanoparticles biosynthesised by Rhodotorula sp. strain ATL72, Egyptian J. Basic Appl. Sci, 5(3), pp. 228 233, DOI: 10.1016/j.ejbas.2018.05.005.

Sowbarnika R., Anhuradha S., Preetha B. (2018). Enhanced antimicrobial effect of yeast mediated silver nanoparticles synthesized from baker's yeast, Int. J. Nanosci. Nanotechnol, 14(1), pp. 33-42.

Tavakoli H., Rastegar H., Taherian M., Samadi M., Rostami H. (2017), The effect of nano-silver packaging in increasing the shelf life of nuts: an in vitro model, Italian Journal of Food Safety, 6, pp. 156-161, DOI: 10.4081/ijfs.2017.6874.

Waghmare S.R., Mulla M.N., Marathe S.R., Sonawane K.D. (2015), Ecofriendly production of silver nanoparticles using Candida utilis and its mechanistic action against pathogenic microorganisms, Biotech, 5, pp. 33-38, DOI: 10.1007/s13205-014-0196-y.

Win T.T., Khan S., Fu P. (2020), Fungus- (Alternaria sp.) mediated silver nanoparticles synthesis, characterization, and screening of antifungal activity against some phytopathogens, $J$. Nanotechnol, 2020, 8828878, DOI: 10.1155/2020/8828878.

Worldwide M.I. (2011), Dynamic light scattering, Common terms defined, Inform white paper; Malwern Instruments Limited: Malvern, UK, pp. 1-6.

Xue B., He D., Gao S., Wang D., Yokoyama K., Wang L. (2016), Biosynthesis of silver nanoparticles by the fungus Arthroderma fulvum and its antifungal activity against genera of Candida, Aspergillus and Fusarium, International journal of nanomedicine, 11, pp. 1899-1906, DOI: 10.2147/IJN.S98339

Yahya S.M., Abdulmumin Y., Abdulmumin T.M., Sagagi B.S., Murtala M., Salau A.K., Hassan S.A. (2021), Biological synthesis, characterization and antimicrobial effect of silver nanoparticles (Ag-NPs) using aqueous extract of mango pulp (Mangifera indica), Journal of Complementary and Alternative Medical Research, 13(4), pp. 39-50, DOI: 10.9734/jocamr/2021/v13i430233.

Zhao X., Wang K., Ai C., Yan L., Jiang C., Shi J. (2021), Improvement of antifungal and antibacterial activities of food packages using silver nanoparticles synthesized by iturin A, Food Packaging and Shelf Life, 28, 100669, DOI: 10.1016/j.fpsl.2021.100669.

Zhou H., Yang D., Mircescu N.E. Ivleva N.P., Schwarzmeier K., Wieser A., Schubert S., Niessner R., Haisch C. (2015), Surface-enhanced Raman scattering detection of bacteria on microarrays at single cell levels using silver nanoparticles, Microchim Acta, 182, pp. 2259 2266, DOI: $10.1007 / \mathrm{s} 00604-015-1570-0$. 